\title{
Corrigendum
}

\section{Corrigenda: Omission of the Description of Informed Consent on the Identifiable Photos and the Description on Ethical Treatment of Experimental Animals}

Editorial Office, Archives of Plastic Surgery

Tel: +82-2-3472-8252

Fax: +82-2-3472-4254

E-mail: apsedit@gmail.com

Out of 52 original articles or case reports published in Archives of Plastic Surgery from January to September 2017, 5 articles lacked the description of informed consent, in which identifiable photos were present. Also, 3 articles lacked the description of ethical treatment of experimental animals. However, the editorial office had already received the informed consent when all submitted manuscripts were arrived at the office. Also, out of 3 articles dealt with experimental animals, it was confirmed that chickens were purchased at a mart in two cases and permission by the animal ethics committee was provided in one case. Therefore, editorial office apologizes readers not checking those ethical issues and publishes corrigendum as follows:

\section{The chicken thigh adductor profundus free muscle flap: A novel validated non-living microsurgery simulation} training model

Georgios Pafitanis, Yasmine Serrar, Maria Raveendran, Ali Ghanem, Simon Myers

2017;44(4):293-300. https://doi.org/10.5999/aps.2017.44.4.293

Note

Chickens used in the experiment were purchased at a mart.

\section{The usefulness of surgical treatment in slow-flow vascular malformation patients}

Gyu Bin Kang, Yong Chan Bae, Su Bong Nam, Seong Hwan Bae, Ji Yoon Sung

2017;44(4):301-307. https://doi.org/10.5999/aps.2017.44.4.301

\section{Note}

The patient provided written informed consent for the publication and the use of their images. 
3. The role of negative-pressure wound therapy in latissimus dorsi flap donor site seroma prevention: A cohort study Apichai Angspatt, Thana Laopiyasakul, Pornthep Pungrasmi, Poonpissamai Suwajo 2107;44(4):308-312. https://doi.org/10.5999/aps.2017.44.4.308

Note PATIENT CONSENT

The patient provided written informed consent for the publication and the use of their images.

\section{Reconstruction of large facial defects via excision of skin cancer using two or more regional flaps}

Dong Min Lee, Yong Chan Bae, Su Bong Nam, Seong Hwan Bae, June Seok Choi

2017;44(4):319-323. https://doi.org/10.5999/aps.2017.44.4.319

Note

PATIENT CONSENT

The patient provided written informed consent for the publication and the use of their images.

\section{Skin necrosis with oculomotor nerve palsy due to a hyaluronic acid filler injection} Jae Il Lee, Seok Joo Kang, Hook Sun

2017;44(4):340-343. https://doi.org/10.5999/aps.2017.44.4.340

Note

PATIENT CONSENT

The patient provided written informed consent for the publication and the use of their images.

\section{Hydrogel and platelet-rich plasma combined treatment to accelerate wound healing in a nude mouse model} Yu Gil Park, In Ho Lee, Eun Soo Park, Jin Young Kim

2017;44(3):194-201. https://doi.org/10.5999/aps.2017.44.3.194

Note

Ethical approval

Before experiment, it was permitted by the Animal Ethics Committee of Bucheon Hospital, Soonchunhyang University (No.: SCHBC-animal-2014-010). Procedure of animal experiment was done based on the animal ethics guideline of the affiliated institute.

\section{Comprehensive analysis of chicken vessels as microvascular anastomosis training model}

Bo Young Kang, Byung-Joon Jeon, Kyeong-Tae Lee, Goo-Hyun Mun

2017;44(1):12-18. https://doi.org/10.5999/aps.2017.44.1.12

Note

Chickens used in the experiment were purchased at a mart.

\section{Contracted nose after silicone implantation: A new classification system and treatment algorithm}

Yong Kyu Kim, Seungho Shin, Nak Heon Kang, Joo Heon Kim

2017;44(1):59-64. https://doi.org/10.5999/aps.2017.44.1.59

Note

PATIENT CONSENT

The patient provided written informed consent for the publication and the use of their images. 